\title{
A new parameterized approach for ionospheric tomography
}

\author{
Biyan Chen ${ }^{1,2,3} \cdot$ Lixin $\mathrm{Wu}^{1,3} \cdot$ Wujiao Dai ${ }^{1,2,3} \cdot$ Xiaomin Luo ${ }^{4} \cdot$ Ying X $^{5}$
}

Received: 18 January 2019 / Accepted: 9 July 2019 / Published online: 15 July 2019

(c) The Author(s) 2019

\begin{abstract}
GNSS ionospheric tomography technique is capable to reconstruct the high-quality 3D ionospheric electron density (IED) images with a relatively low cost. We present a new parameterized approach for refining the voxel-based ionospheric tomography modeling. This approach is different to most other voxel-based techniques as they assume a homogeneous IED distribution in each voxel that is unreasonable for the tomography modeling. In this method, IED of any point within a voxel is determined via vertically exponential interpolation and horizontally inverse distance weighted interpolation from the IED values at the eight corners of that voxel. The parameterized tomography is tested with real data collected over the period of June 1-30, 2015 from 45 GPS stations in south China. The superiority of the new parameterized method is verified by comparison with the traditional nonparametric method. The new parameterized method outperforms the traditional method by $12 \%, 10 \%, 5 \%$ and $2 \%$ for vertical resolutions of $25 \mathrm{~km}, 50 \mathrm{~km}, 75 \mathrm{~km}$ and $100 \mathrm{~km}$, respectively, in the self-consistency validation by GPS data. Such improvements are 20\%, 24\%,22\% and 16\%, respectively, when assessed by the Swarm in situ IEDs. In terms of the vertical layer discretization, configurations using the resolution of $25 \mathrm{~km}$ generally performs better than the other three vertical resolutions. Overall, the parameterized method using a vertical resolution of $25 \mathrm{~km}$ achieves the best performance from the comprehensive comparisons with ionospheric data derived by GPS, ionosonde and Swarm satellites.
\end{abstract}

Keywords Parameterized ionospheric tomography · Ionospheric electron density (IED) · Total electron content (TEC) · Ionosonde $\cdot$ Swarm

Biyan Chen

yeary124@csu.edu.cn

$\triangle$ Xiaomin Luo

xmluo@whu.edu.cn

1 School of Geosciences and Info-Physics, Central South University, Changsha, China

2 Key Laboratory of Metallogenic Prediction of Nonferrous Metals and Geological Environment Monitoring Ministry of Education, School of Geosciences and Info-physics, Central South University, Changsha, Hunan, China

3 Key Laboratory of Precise Engineering Surveying and Deformation Disaster Monitoring of Hunan Province, Changsha, Hunan, China

4 GNSS Research Centre, Wuhan University, Wuhan, China

5 College of Geomatics, Shandong University of Science and Technology, Qingdao, China

\section{Introduction}

The ionosphere, stretching from the height of about $50 \mathrm{~km}$ to several $1000 \mathrm{~km}$ above the earth surface, is filled with free electrons, electrically charged molecules and atoms which are primarily generated by the ultraviolet radiation from the sun (Komjathy 1997; Bidaine and Warnant 2010). The presence of free electrons in the ionosphere can exert adverse effects on various communication, surveillance and navigation systems (Bust and Mitchell 2008). In the study of seismo-ionospheric coupling, ionospheric electron density (IED) anomalies have been frequently detected before the earthquakes (Pulinets and Boyarchuk 2004), offering a critical means in the detection of a pre-earthquake anomaly. Therefore, the imaging of the IED field is central to various fields including space weather studies (He et al. 2018), and geophysical, space geodetic and radiophysical applications (Yang et al. 2017).

The technique of ionospheric tomography can reconstruct the IED images through the use of slant total electron content (TEC) measurements along different rays penetrating the 
model space from various directions. The first research work was published in 1986 when Austen et al. (1986) applied the tomographic method to reconstruct 2D IED images using the scan of the ionosphere came from a polar-orbiting satellite. However, due to the technical and instrumental limitations, the tomographic IED profiles are not necessarily obtained at any time and place around the world, greatly degrading its usability in practical applications (Ma 2005). The advent of the global positioning system (GPS) enables tomography to map the 3D IED structures with advantages of high precision, all-weather capability and near-real-time operability (Bust 2004; Zheng et al. 2015; Yang et al. 2017; Jin and Li 2018). A large amount of significant works thus has been carried out regarding to theoretical models and experimental analysis for GPS-based ionospheric tomography (Ruffini et al. 1998; Kersley 2005; Jin and Park 2007; Yao et al. 2013a; Alizadeh et al. 2015; Minkwitz et al. 2015; dos Santos Prol and de Oliveira Camargo 2015; Minkwitz et al. 2016).

In general, two different classes of models can be applied to perform the ionospheric tomography: one is the functionbased ionospheric model (Hansen et al. 1997; Ruffini et al. 1998; Gao and Liu 2002; Nesterov and Kunitsyn 2011); and another one is the voxel-based ionospheric model (Rius et al. 1997; Ma 2005; Wen et al. 2008; Zheng et al. 2015; Jin and Li 2018). The function-based model adopts a set of functions to express IED distribution with the advantage of using very few parameters to describe a large ionospheric region. But subject to the geometry defined by the distribution of the ground GNSS stations and the satellite constellation, it is very difficult to solve tomographic equations if we directly fit the observations (Yao et al. 2013a). For the voxel-based model, the ionosphere is normally discretized into several voxels assuming that the IED in each voxel is constant and evenly distributed. During a tomographic period (e.g., 1 h), however, it is mostly not possible to have enough GNSS satellites and ground stations to allocate enough slant TEC (STEC) measurements for each voxel. Thus, the voxel-based tomography yields an ill-posed problem, and many voxels are underdetermined.

Due to the simple operation and easy solution, the voxelbased model is often exploited in ionospheric tomography studies (Wen et al. 2012; Yang et al. 2017; Zheng et al. 2017; Jin and Li 2018). To circumvent the ill-posed problem, many algorithms have been developed over the years. Based on 3-D variational data assimilation technique, Bust (2004) developed the Ionospheric Data Assimilation Three Dimensional (IDA3D) algorithm which adopts an exponential time covariance model to predict the IED state vector and its covariance matrix from one time step to the next. The determination of the covariance matrix of the state vector and the right choice of the time prediction model are important for this approach (Minkwitz et al. 2015). Wen et al. (2012) proposed a two-step algorithm for ionospheric tomography solution by jointly using the Phillips smoothing method (also known as Tikhonov regularization) and the multiplicative algebraic reconstruction technique (MART). Experimental results show that the new method performed better in both numerical simulations and practical applications. Yao et al. (2014) proposed a 3D iterative reconstruction algorithm based on the minimization of total variation. The authors presented the significant improvements of the proposed algorithm in IED reconstruction under both quiescent and disturbed conditions. Seemala et al. (2014) applied the constrained least squares fit to reconstruct the IED distributions using GPS Earth Observation Network (GEONET) in Japan. Independent of the initial guess from a model, the proposed method uses a restrain parameter derived from the NeQuick model to constrain the IED profile in the tomography. Zheng et al. (2015) reported a multi-scale ionospheric tomography which parameterizes the model with overlapping pixels of different sizes. They showed that the multiscale model could bring about a 15\% improvement in accuracy compared with the conventional single-scale model. Jin and Li (2018) developed an improved two-step algorithm, in which the NeQuick 2 is used to improve the a priori model of International Reference Ionosphere (IRI) 2012, and then the MART is implemented to obtain the final IED distribution. Based on the GNSS Earth Observation Network (GEONET) observations of Japan, assessments by ionosonde and radio occultation data demonstrated the feasibility and superiority of their new method. Most recently, Norberg et al. (2018) reported the use of Gaussian Markov random field priors for tomographic solution based on Bayesian statistical inversion. The method can provide physically quantifiable probabilistic interpretation for all model variables, as well as easing computational burden (Norberg et al. 2015, 2018).

However, a critical deficiency in the voxel-based model is the assumption of a homogeneous distribution of IED in each voxel, which was widely used in previous studies. The IEDs vary a lot within the voxel space, especially in the vertical direction. Higher resolution may mitigate the adverse effects brought by the improper assumption, but it will increase the computational costs and the influence of inter voxel constraints on the results. As early as 1984, Andersen and Kak (1984) proposed an improved Simultaneous Algebraic Reconstruction Technique (SART) which uses bilinear elements for discrete approximation to the ray integrals of a continuous image. The ray integral was approximated by a finite sum involving a set of equidistant points along that ray, while the point value was determined by bilinear interpolation from four neighboring points of the sampling grid. This work indicated that continuous image representation using bilinear elements performed better than traditional pixel-based method for the 2D image reconstruction. In the field of GNSS tomography, 
similarly, Perler et al. (2011) proposed to parameterize the water vapor tomography model by expressing the wet refractivity of each point using trilinear or spline functions from its eight neighboring voxel nodes. They pointed out that the parameterization of voxels is an effective way to reduce the effects of unreasonable discretization and verified its superiority in various tests. To our knowledge, however, almost no studies have reported the application of the parametrized method in ionospheric tomography. In addition, bilinear/spline methods were used in the interpolation without considering the vertical variation characteristic of the wet refractivity (Perler et al. 2011). Therefore, based on Perler et al. (2011), we develop a new parameterized approach to refine the ionospheric tomography model, aiming to improve the inversion accuracy of IED reconstruction. In this method, IED within a voxel varies with space and is determined from the IED values at the eight corners of that voxel by considering the spatial variation characteristics of the IED. Similar with the function-based model, this parameterized approach describes the IED fields in a consecutive way, while inheriting the merits of the voxel-based model.

The methodology of the parameterized ionospheric tomography is described first. Thereafter, we present the datasets used to perform the tomography experiment and the validation results of the parameterized tomography method. STECs measured by GPS and IEDs derived from ionosonde and Swarm satellites are adopted to evaluate the performance of the tomographic solutions. Finally, a summary of this study is given in the last section.

\section{Modeling the IED field with parameterized ionospheric tomography}

Along the ray path from a receiver to a GNSS satellite, the relationship between the STEC and IED can be expressed as:

$\mathrm{STEC}=\oint_{l} N_{e} d_{l}$

where $N_{e}$ is the ionospheric electron density along the ray path $l$ of the signal through the ionosphere. Ionospheric tomography uses a series of STECs interweaving in the space across various directions to inverse the spatial distribution of IED. The voxel-based model must discretize the reconstruction space into a number of voxels (Fig. 1). As a simple case shown in Fig. 1, the space is divided into four voxels with their numbers marked in blue. In the traditional nonparametric method (hereafter short for traditional method), it is assumed that IED is invariable and evenly

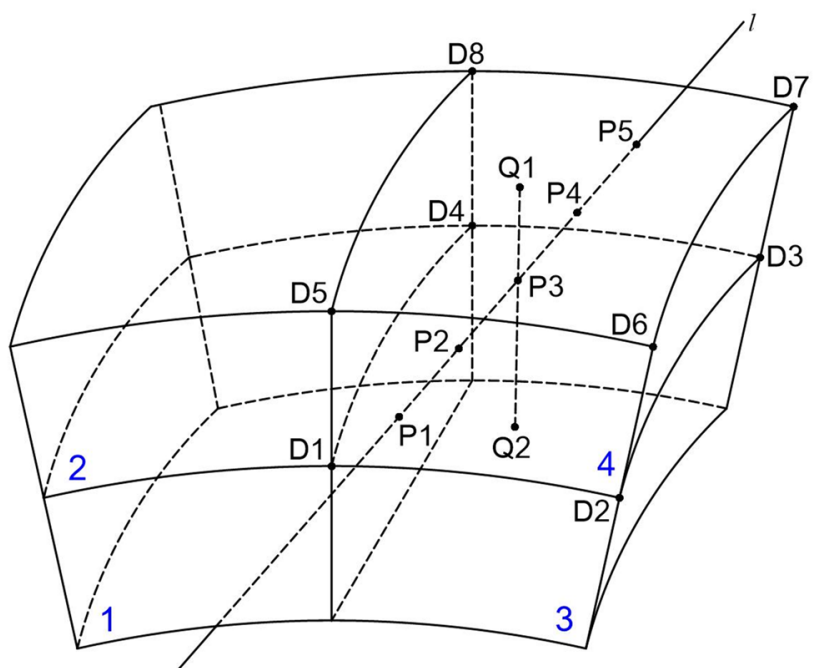

Fig. 1 Schematic representation of voxel discretization for ionospheric tomography model

distributed within each voxel during the reconstruction period. Thus, each STEC can be regarded as a summation of all segments that cross through those voxels along the ray path. Then (1) can be approximated by:

$\mathrm{STEC}=\sum_{i=1}^{n} N_{e}^{i} d_{i}$

where $n$ is the number of voxels crossed by the STEC, $N_{e}^{i}$ refers to the electron density in the voxel $i$, and $d_{i}$ is the intercept of the STEC ray path by the voxel $i$.

In the parameterized model, the IED within each voxel is no longer assumed be constant whereas it changes with the position. The IED at any point within a voxel is determined by a weighted sum of the eight IED values at the corners of that voxel. This allows the STEC to be expressed as an integral of the IEDs at the voxel nodes. Since the integral cannot be expressed analytically in most cases, the Newton-Cotes quadrature is adopted to solve the integrals (Perler et al. 2011). As shown in Fig. 1, the integral of the IEDs along P1-P5 can be solved by:

$$
\begin{aligned}
\int_{\mathrm{P} 1}^{\mathrm{P} 5} N_{e} \mathrm{~d} l= & \frac{\mathrm{P} 5-\mathrm{P} 1}{90}\left\{7\left[N_{e}(\mathrm{P} 1)+N_{e}(\mathrm{P} 5)\right]\right. \\
& \left.+32\left[N_{e}(\mathrm{P} 2)+N_{e}(\mathrm{P} 4)\right]+12 N_{e}(\mathrm{P} 3)\right\}
\end{aligned}
$$

where $\mathrm{P} i=\mathrm{P} 1+\frac{\mathrm{P} 5-\mathrm{P} 1}{4}(i-1)$. The IED of point $\mathrm{P} i$ is determined by the IED values of the eight voxel nodes, i.e. $D_{1}, D_{2}, \ldots, D_{8}$. P1 and P5 are located at the voxel boundaries, while $\mathrm{P} 2, \mathrm{P} 3$, and $\mathrm{P} 4$ are located within the voxel. P1 is located at the surface defined by $D_{1}, D_{2}, D_{3}, D_{4}$ and $\mathrm{P} 5$ is located at the surface defined by $D_{5}, D_{6}, D_{7}$ and $D_{8}$. Taking 
P3 as an example, its IED value can be vertically interpolated with points $Q_{1}$ and $Q_{2}$ :

$$
\begin{aligned}
N_{e}(\mathrm{P} 3)= & \frac{h_{\mathrm{P} 3}-h_{Q_{2}}}{\left(h_{Q_{1}}-h_{Q_{2}}\right) \cdot e^{\alpha\left(h_{Q_{1}}-h_{\mathrm{P} 3}\right)}} N_{e}\left(Q_{1}\right) \\
& +\frac{h_{Q_{1}}-h_{\mathrm{P} 3}}{h_{Q_{1}}-h_{Q_{2}}} e^{\alpha\left(h_{\mathrm{P} 3}-h_{Q_{2}}\right)} N_{e}\left(Q_{2}\right)
\end{aligned}
$$

$Q_{1}$ and $Q_{2}$ are located at the upper and bottom boundaries of voxel 4 , respectively. $\alpha$ is a parameter which can be estimated from the following expression:

$N_{e}\left(h_{i}\right)=N_{e}\left(h_{0}\right) e^{\alpha\left(h_{i}-h_{0}\right)}$

where $h_{0}$ refers to the altitude of the lower surface of the voxel and $h_{i}$ refers to the altitude of any point within the voxel. The (5) is established given the exponential variation of IED with the altitude (Alizadeh et al. 2015). To improve the modeling performance, $\alpha$ is estimated for each vertical layer using IRI 2016 profiles. In addition, the IED values of $Q_{1}$ and $Q_{2}$ are calculated using the inverse distance weighted interpolation (Ding et al. 2017):

$N_{e}\left(Q_{i}\right)=\frac{\sum_{i=1}^{4} d_{i}^{-1} \cdot N_{e}\left(D_{i}\right)}{\sum_{i=1}^{4} d_{i}^{-1}}$

where $N_{e}\left(D_{i}\right)(i=1,2,3,4)$ are the four surrounding nodes of the voxel surface, in which the point $Q_{i}$ is located.

Stacking all the STECs during the reconstruction period (60 min in this study), a linear system between the STECs and the IED field is established:

$\mathbf{y}=\mathbf{A x}$

where $\mathbf{y}$ is the vector of STEC measurements, $\mathbf{x}$ is the unknown parameter vector containing the IED of all voxel nodes, and $\mathbf{A}$ is the design matrix composed of the contributions of $\mathbf{x}$ on the STEC measurements.

The A matrix of (7) is often not squared, ill-posed and ill-conditioned. Thus generalize inverse has to be performed. Many methods have been employed to circumvent the problem of ill-posedness in GNSS tomographic equation, such as iterative algorithms (ART, Algebraic Reconstruction Technique) (Bender et al. 2011; Yao et al. 2014; Zheng et al. 2015; Yang et al. 2017; Jin and Li 2018), classical constrained solution (Hirahara 2000), Singular Value Decomposition (Bhuyan et al. 2002; Wen et al. 2008), Kalman filter approach (Nilsson and Gradinarsky 2006; Perler et al. 2011), or regularization method (Wen et al. 2012; Yao et al. 2013b; Wang et al. 2016). Among the various methods, an iterative approach is often adopted for solving the ionospheric tomographic equations due to its advantage to avoid time-consuming matrix operations. The iterative approach is able to reconstruct the IED images with high numerical stability and computational efficiency even under bad conditions (Bender et al. 2011). In the comparison with other algorithms of the ART family, Bender et al. (2011) found that the MART can provide the best results with least processing time. Thus, MART is often used to resolve the ill-posed equations of the GNSS tomography (Wen et al. 2012; Chen and Liu 2014; dos Santos Prol and de Oliveira Camargo 2015; Yang et al. 2017). Hence, we perform the inversion of system (7) using MART to attain unknowns. For the $k$ th iteration, the ratio between the observed $\mathbf{y}$ and reconstructed $\mathbf{A}, \mathbf{x}^{k-1}$ is calculated to derive corrections for voxel nodes. Specifically, the correction for the $j$ th voxel node from the $i$ th ray in the $k$ th MART iteration is given by the following formula:

$x_{j}^{k}=x_{j}^{k-1} \cdot\left(\frac{\mathbf{y}_{i}}{\mathbf{A}_{i}, \mathbf{x}^{k-1}}\right)^{\frac{\lambda \mathrm{A}_{i j} x_{j}^{k-1}}{\sum_{j=1}^{n} \mathrm{~A}_{i j} j_{j}^{k-1}}}$

where $\lambda$ is the relaxation parameter with an empirical value of 0.9 used in our study. The IED field provided by the IRI 2016 is adopted as initial value for the iteration. Since a number of voxels are not crossed by any rays, both horizontal and vertical constraints are imposed to the tomographic equation. Horizontal and vertical constraints according to (4) and (6) are used to complement the voxel nodes without any correction in (8). The constraints work as a regularization for the ill-posed problem as more voxels are involved with each measurement.

To make a direct comparison with the parameterized method, the tomographic solutions are performed using the traditional method. Horizontal and vertical constraints are also used in the traditional tomography but differ with the parameterized method. In the horizontal direction, IED of a voxel is assumed to be equal to the inverse distance weighted interpolation from the IED values of its surrounding voxels. Vertically, the IED is assumed to follow an exponential distribution with the variation parameter derived from the IRI 2016 model. Different from the traditional method, the solved unknowns from the parameterized method are IEDs of the voxel nodes. Thus, to get the IED of any point, vertical interpolation in (4) and horizontal interpolation in (6) need to be performed using the IED values of the eight voxel nodes where the point is located. Whereas in the traditional tomography model any point's IED is equal to the IED value of its located voxel. 
Fig. 2 Geographic distribution of GPS stations (red pentagrams) in south China adopted in the ionospheric tomography study. The three black triangles represent GPS stations used for self-consistency validation. The blue diamond indicates the location of the ionosonde station

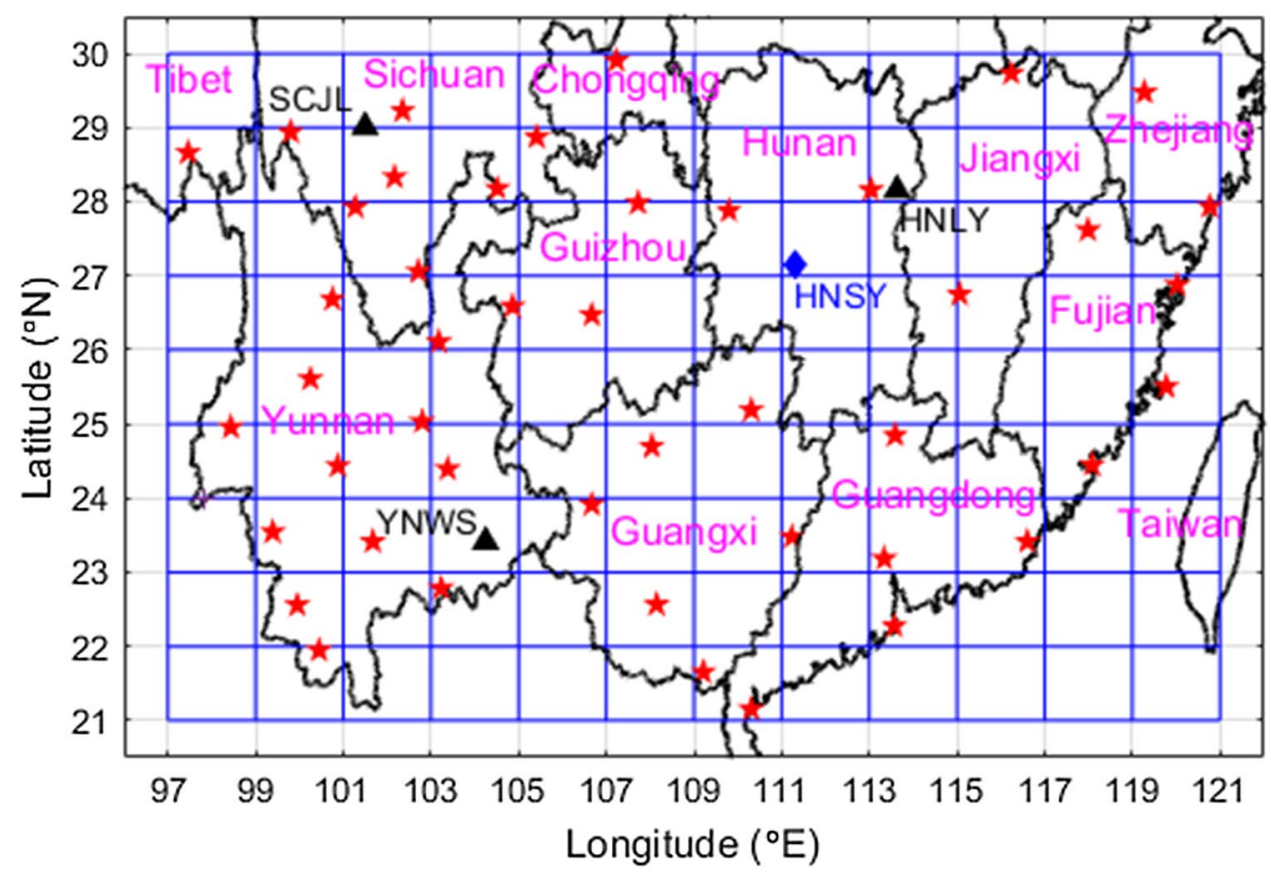

\section{Validation of parameterized tomography model}

This section presents the validation results and discussion of the performance of parameterized ionospheric tomography in comparison with the GPS, ionosonde and Swarm measurements. In this study, 45 GPS stations (Fig. 2) from the Crustal Movement Observation Network of China (CMONOC) are used in the ionospheric tomography experiment. In addition, STECs from stations HNLY, SCJL and YNWS (black triangles shown in Fig. 2) are used for self-consistency validation purposes and are not included in the input dataset. The tomographic region covers from $97 \mathrm{E}$ to $121 \mathrm{E}$ in longitude, $21 \mathrm{~N}-30 \mathrm{~N}$ in latitude and $100-1000 \mathrm{~km}$ in altitude, respectively. Since the IED variations in latitude are greater than in longitude, grid intervals are set as 2 and 1, respectively. In the altitude, four different layer discretization intervals, i.e., $25 \mathrm{~km}$, $50 \mathrm{~km}, 75 \mathrm{~km}$ and $100 \mathrm{~km}$, are employed to examine their impacts on tomographic solutions. To examine the possible benefits to ionospheric tomography brought by the parameterized method, experiments using traditional method are also performed for a direct comparison. For the sake of simplicity, abbreviations of Trad_25, Trad_50, Trad_75 and Trad_100 represent the traditional tomography using the vertical resolution of $25 \mathrm{~km}, 50 \mathrm{~km}, 75 \mathrm{~km}$ and $100 \mathrm{~km}$, respectively; and Para_25, Para_50, Para_75 and Para_100 represent the parameterized tomography using the vertical resolution of $25 \mathrm{~km}, 50 \mathrm{~km}, 75 \mathrm{~km}$ and $100 \mathrm{~km}$, respectively. GPS data collected over the whole month of June of 2015 are used. In addition, IED profiles provided by ionosonde station HNSY (blue diamond in Fig. 2), and Swarm measured in situ IEDs are employed to validate the tomographic solutions.

\section{Self-consistency validation by GPS}

To perform the self-consistency validation, the three GPS stations HNLY, SCJL and YNWS were not included in the ionospheric tomography experiments. STEC measurements derived from the GPS observations over the period of June
Table 1 Statistical results of the differences between STECs measured by GPS and STECs derived from IRI 2016 and tomographic solutions using traditional and parameterized methods

\begin{tabular}{|c|c|c|c|c|c|c|c|c|c|}
\hline \multirow[t]{2}{*}{ Statistics } & \multirow[t]{2}{*}{ IRI2016 } & \multicolumn{4}{|c|}{ Traditional method } & \multicolumn{4}{|c|}{ Parameterized method } \\
\hline & & Trad_25 & Trad_50 & Trad_75 & Trad_100 & Para_25 & Para_50 & Para_75 & Para_100 \\
\hline Bias & 1.22 & 1.21 & 1.09 & 1.00 & 0.78 & 1.06 & 1.11 & 1.04 & 0.84 \\
\hline RMS & 8.21 & 3.23 & 3.34 & 3.38 & 3.39 & 2.84 & 3.02 & 3.20 & 3.32 \\
\hline Min & -24.58 & -10.65 & -11.31 & -10.34 & -10.52 & -8.27 & -8.54 & -8.87 & -9.30 \\
\hline Max & 27.41 & 13.08 & 13.28 & 13.34 & 14.33 & 11.48 & 12.15 & 12.55 & 12.62 \\
\hline
\end{tabular}

Statistics are derived from the three GPS stations over the whole month of June 2015 (unit: TECU) 
1-30, 2015 are directly compared with those calculated from the IRI 2016 model and tomographic results of the corresponding epochs. Table 1 gives the statistics of the comparison results. STEC derived IRI 2016 model performs badly as its root mean square (RMS) error of 8.21 TECU is almost three times that of the tomographic scheme Para 25. In terms of mean bias, both the traditional and parameterized methods yield consistently positive values for the four different vertical resolution schemes. This is probably due to the contribution of the plasmasphere to the GPS STECs as the space above the altitude of $1000 \mathrm{~km}$ is ignored in the ionospheric tomography model. For the RMS errors, the parameterized method obtains smaller values for all the four vertical resolution schemes compared with the traditional method. The improvements achieved by the parameterized method are $12 \%, 10 \%, 5 \%$ and $2 \%$ for vertical resolutions of $25 \mathrm{~km}, 50 \mathrm{~km}, 75 \mathrm{~km}$ and $100 \mathrm{~km}$, respectively. In addition, we can notice that in both methods the accuracies of tomographic STECs decrease with the increase in the vertical layer height. The best performance is obtained by the Para_25 with an RMS error of 2.84 TECU. We also examined the performance of the vertical resolution of $15 \mathrm{~km}$, however, no further improvements are obtained.

Figure 3 further shows the performance of the tomographic solutions at the three GPS stations. The RMS errors vary in the range of 2.5-3.7 TECU for the eight different tomography schemes for the three GPS stations. For each station, in general, the performance of the tomography degrades with the increase in the vertical layer height. Also, the tomography scheme of Para 25 consistently achieves the highest accuracy at all the three GPS stations. In addition, tomographic STECs have the best and worst agreements with the GNSS measured ones at stations SCJL and HNLY, respectively. This is due to the different GPS networks at the corresponding GPS stations. As observed from Fig. 2, the GPS network at the HNLY is much sparser than those at the SCJL and YNWS stations. The denser the ground-based GPS network, the better performance of the tomography will be obtained. Therefore, the tomographic solutions perform worst at station HNLY.

\section{Validation of IED profiles by ionosonde}

It should be noted that although the STECs derived from tomographic IED fields agree well with those from the GPS, it does not guarantee that the IED vertical profiles have been correctly modeled. Therefore, in this section, the tomographic IED profiles are further compared with those observed by the ionosonde. An ionosonde probes the ionospheric structure by broadcasting a sweep of high frequencies, usually in the range of 0.1 to $30 \mathrm{MHz}$ (Reinisch et al. 2009). Due to its high accuracy, the ionosonde-derived IED profiles have been often employed as ground truth reference to validate alternative ionospheric techniques, e.g., tomography (Zheng et al. 2017) and radio occultation (Habarulema and Carelse 2016). As shown in Fig. 2, one ionosonde station HNSY (111.28 E, $27.14 \mathrm{~N}, 62 \mathrm{~m})$ is located in the northeast-central part of the tomographic region. This station can provide the IED profiles up to an altitude of $1000 \mathrm{~km}$, which are very useful to validate the tomographic solutions. Here, one should note that the ionosonde can only measure the IED profiles below the $F$ region peak (around $350 \mathrm{~km}$ ). The
Fig. 3 RMS errors of the reconstructed STECs from IRI 2016 and by using four different vertical resolution schemes with the traditional and parameterized tomography at GPS stations HNLY, SCJL and YNWS

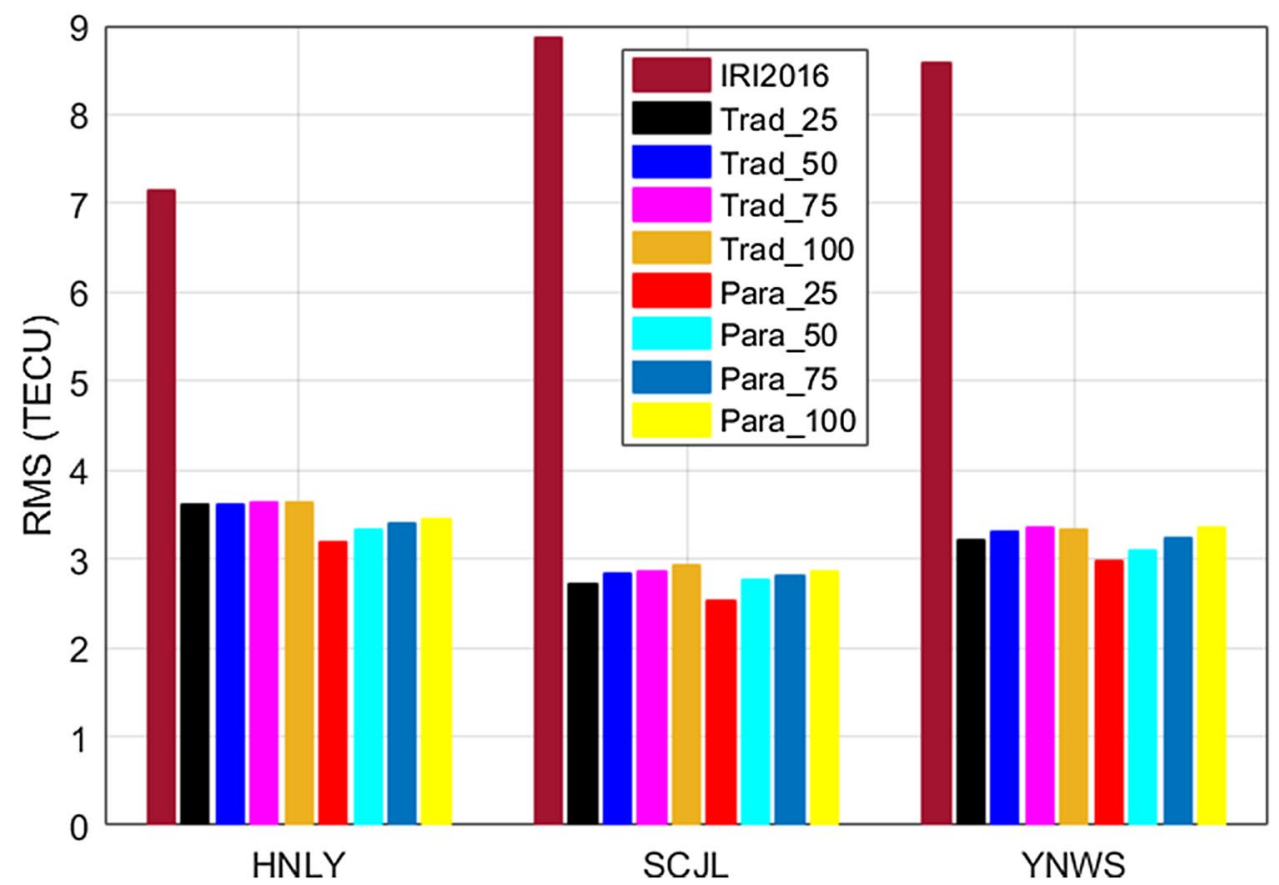


Fig. 4 IED profiles derived from IRI 2016, ionosonde and tomography. Time periods in left (UT 07:00-08:00 on June 5, 2015) and right (UT 05:0006:00 on June 23, 2015) panels correspond to the occurrences of lowest (0) and highest (8+) $\mathrm{Kp}$-index, respectively

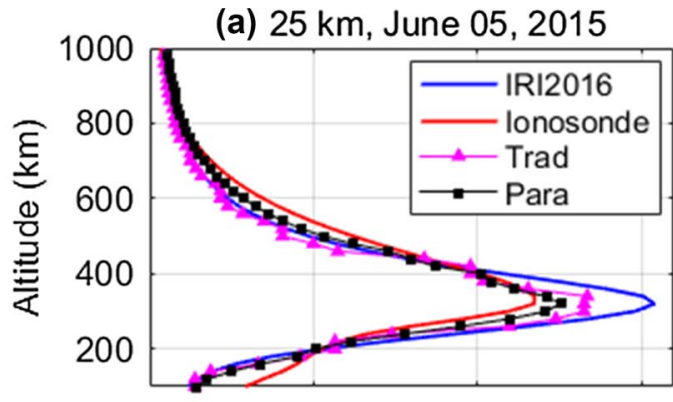

(b) $50 \mathrm{~km}$, June 05, 2015

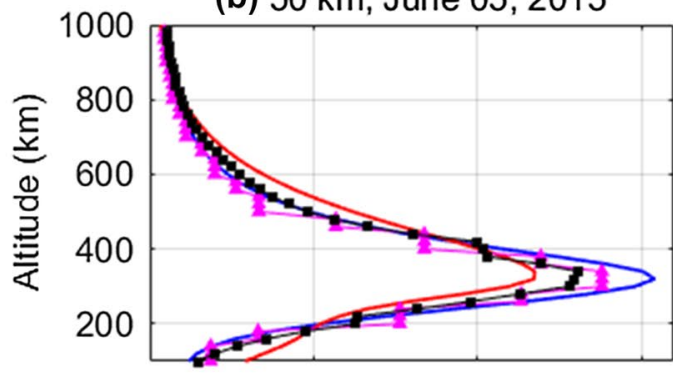

(c) $75 \mathrm{~km}$, June 05, 2015

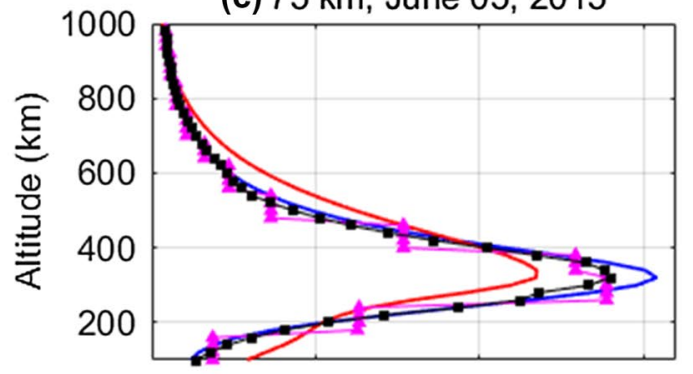

(d) $100 \mathrm{~km}$, June 05, 2015

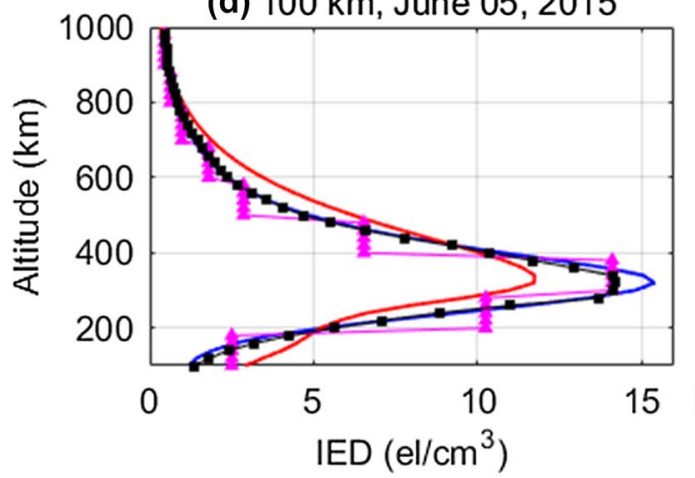

(e) $25 \mathrm{~km}$, June 23,2015

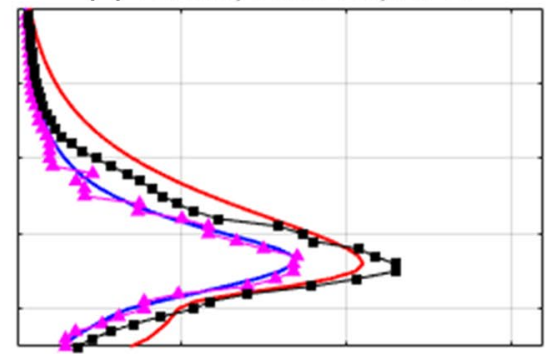

(f) $50 \mathrm{~km}$, June 23, 2015

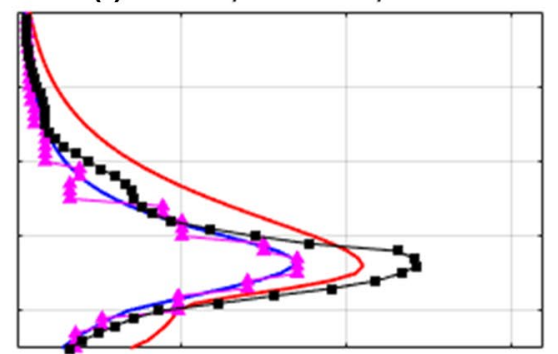

(g) $75 \mathrm{~km}$, June 23, 2015

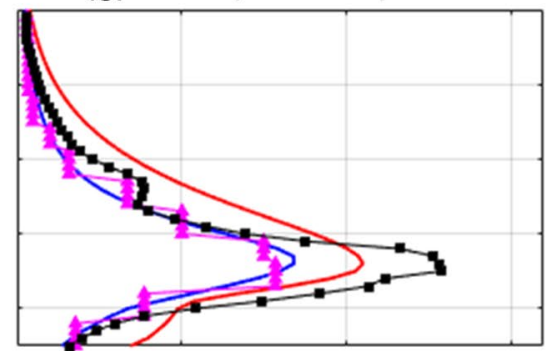

(h) $100 \mathrm{~km}$, June 23, 2015

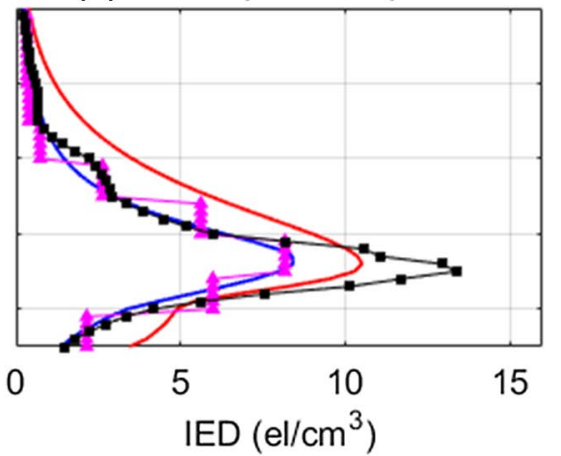

top-side profiles of ionosonde are extrapolated from a model and thus not very reliable for validation purposes. To make a direct comparison, the IEDs from the ionosonde and the tomography are interpolated to heights of $100-1000 \mathrm{~km}$ with an interval of $20 \mathrm{~km}$, according to the interpolation method described in (4). Matchup IEDs of the traditional method are obtained by searching the voxel where the point is located. The matchup IED values are interpolated according to (4) and (6) for the parameterized method.

Figure 4 shows comparisons between IRI 2016, ionosonde and tomography at two periods, 07:00-08:00 UT on
June 5, 2015 (left panels) and 05:00-06:00 UT on June 23, 2015 (right panels). These two periods are selected since they correspond to the minimum and maximum geomagnetic activities during the 30 test days. Panels (a) and (e), (b) and (f), (c) and (g) and (d) and (h) present the comparisons for vertical resolutions of $25 \mathrm{~km}, 50 \mathrm{~km}, 75 \mathrm{~km}$ and $100 \mathrm{~km}$, respectively. We can see that the tomography can improve the initial IED fields provided by IRI 2016 model significantly as tomographic results are closer to ionosonde profiles. In the left panels $(\mathrm{a}-\mathrm{d})$, the tomographic IED profiles from all the eight schemes generally agree with the 
ionosonde profile. However, large discrepancies are exhibited at most altitudes in the right panels $(\mathrm{e}-\mathrm{h})$, perhaps due to the influence of strong geomagnetic activity. In both comparisons, the IED profiles derived from the parameterized method generally agree better with the ionosonde than those solved by the traditional method. Among them, IED profiles of Para 25 show the best agreement with ionosonde profiles in both comparisons (Fig. 4a and e). For the traditional method, the IED value of any point is same as that of the voxel, where the point is located. This is the reason why in Fig. 4 many IED values from the traditional method show no changes with height. This phenomenon further exhibits the drawback of the traditional method.
IED sections along the longitude of $111.28 \mathrm{E}$ and the latitude of $27.14 \mathrm{~N}$ for the above two periods are further displayed in Figs. 5 and 6, respectively. The longitude and latitude correspond to the location of the ionosonde. Since the vertical resolution of $25 \mathrm{~km}$ achieves the best performance in general, we only present the tomographic profiles from Trad 25 and Para 25 for a comparison with IRI model. As shown in the figures, IEDs provided by IRI 2016 model (top panels) vary smoothly in the space. However, obvious disturbances can be observed in altitudes of 200-500 km of IED profiles derived from tomography (middle and bottom panels), especially during the period of 05:00-06:00 UT on June 23, 2015 (right panels). This demonstrates the capability
Fig. 5 IED sections along the longitude of 111.28 E for periods of UT 07:00-08:00 on June 5, 2015 (left panels) and UT 05:00-06:00 on June 23, 2015 (right panels). IED profiles are derived from IRI 2016 model (top panels), and tomography using schemes Trad_25 (middle panels) and Para_25 (bottom panels)
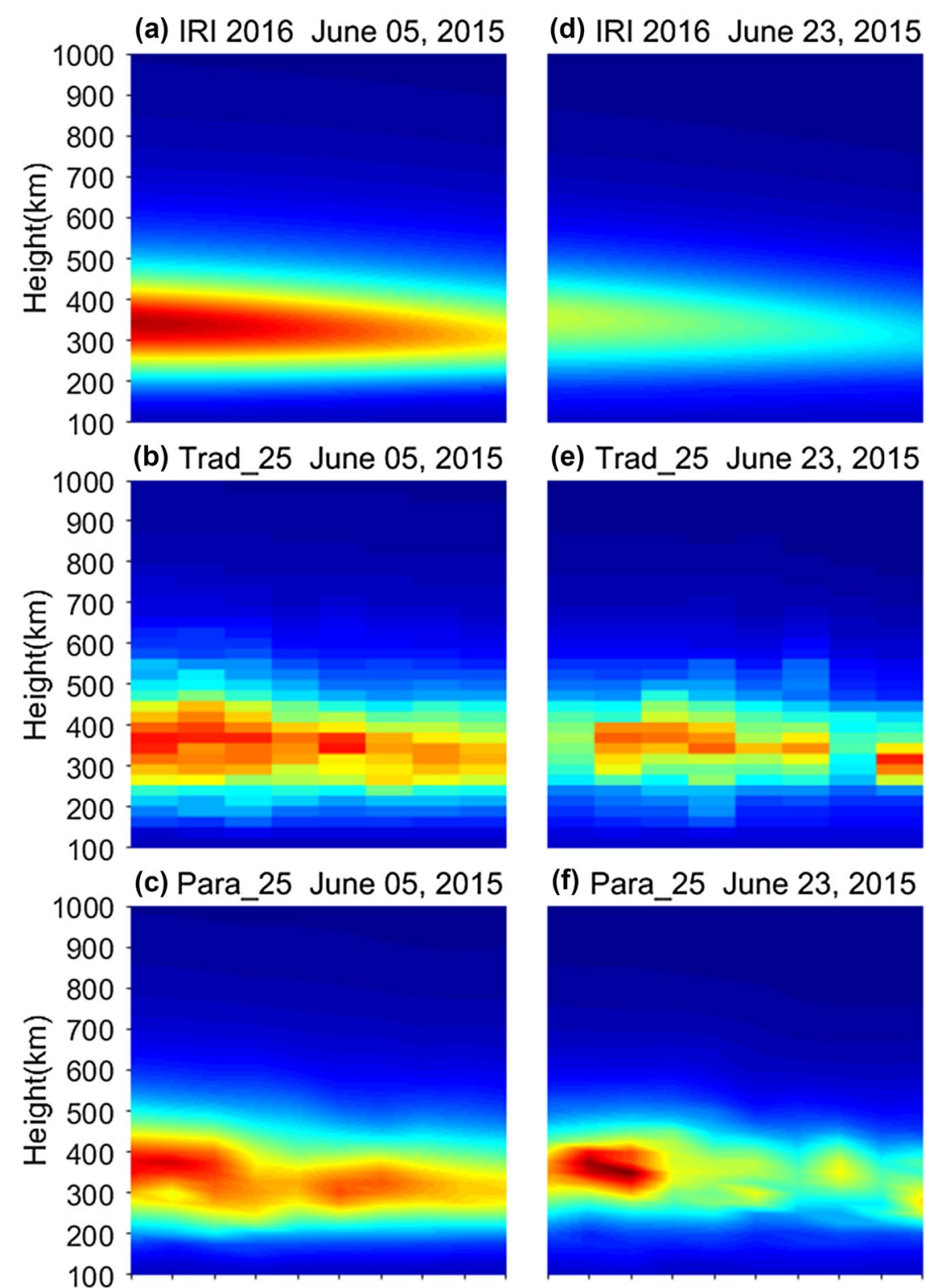

(e) Trad_25 June 23, 2015

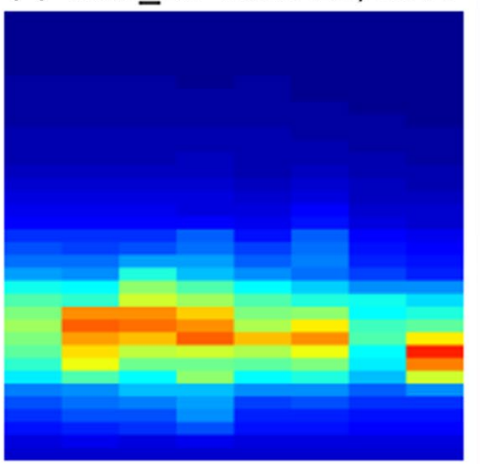

1.2
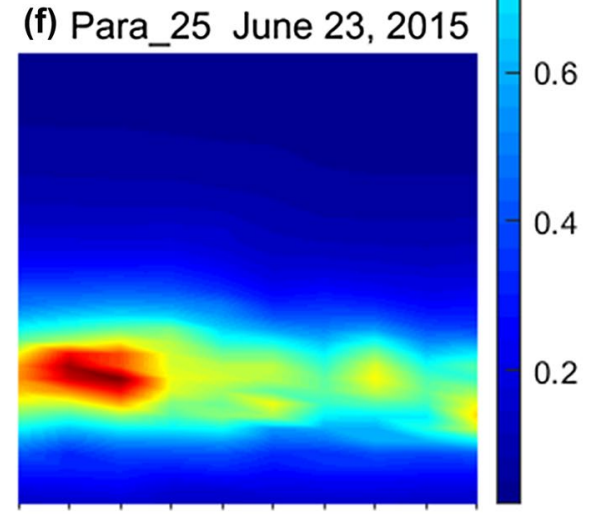

2122232425262728293021222324252627282930 Latitude $\left({ }^{\circ} \mathrm{N}\right)$ 
Fig. 6 IED sections along the latitude of $27.14 \mathrm{~N}$ for periods of UT 07:00-08:00 on June 5, 2015 (left panels) and UT 05:00-06:00 on June 23, 2015 (right panels). IED profiles are derived from IRI 2016 model (top panels), and tomography using schemes Trad_25 (middle panels) and Para_25 (bottom panels)
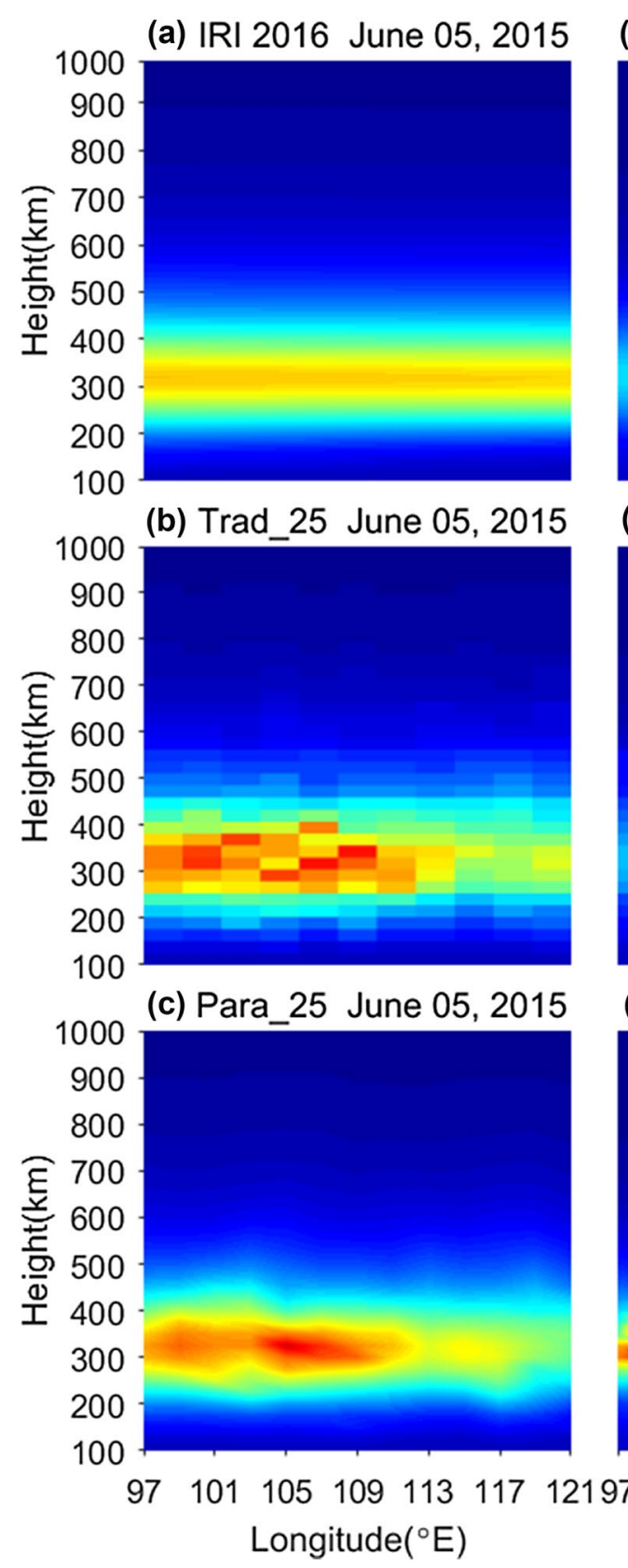

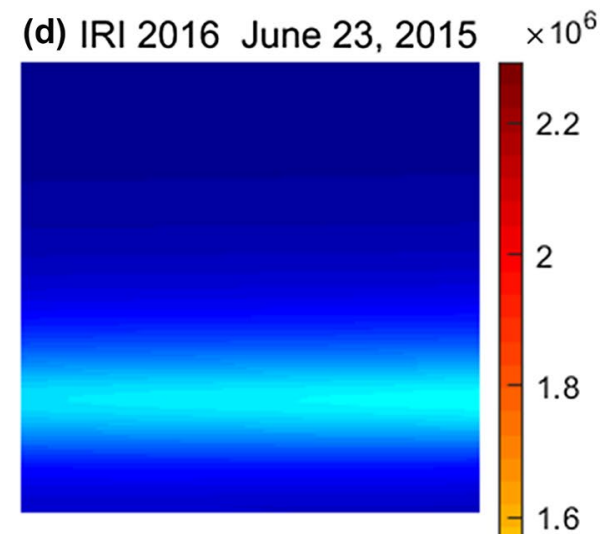

(e) Trad_25 June 23, 2015

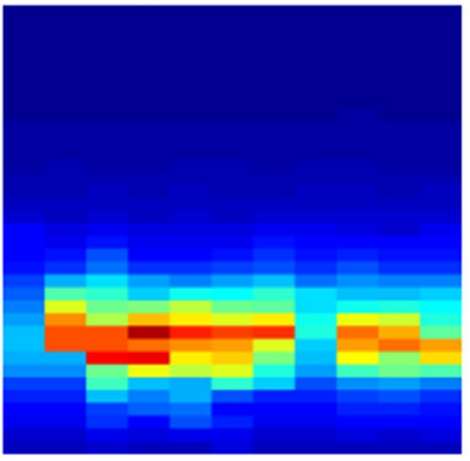

1.4

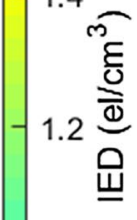

(f) Para_25 June 23, 2015

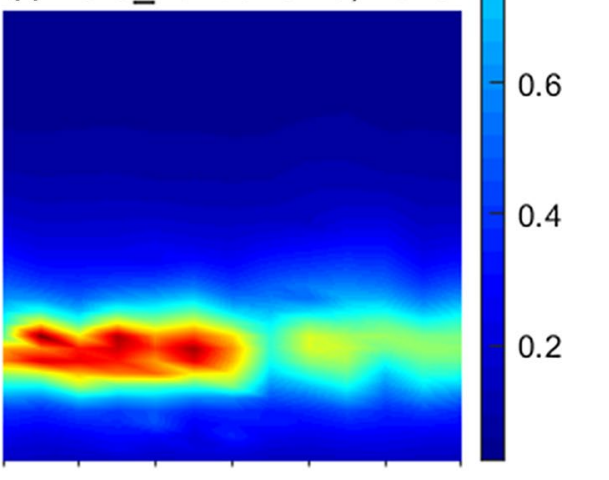

$101105109113 \quad 117 \quad 121$

Longitude $\left({ }^{\circ} \mathrm{E}\right)$ of tomography in monitoring ionospheric disturbances. As shown in the figures, due to the assumption of the homogeneous distribution of IED in each voxel, IED images generated by traditional method (middle panels) show a mosaic pattern. The mosaic-like images fail to clearly depict the IED distribution as good as the parameterized approach (bottom panels). The benefit to ionospheric tomography brought by the parameterized approach can be judged intuitively from the comparative analysis of the IED images.

To study the performance of tomographic solutions at different layers, the RMS errors and the relative RMS errors of the differences between ionosonde and tomography at different altitudes are calculated. The relative RMS is defined as the ionosonde measured IED divided by the RMS. Figure 7 displays the change of RMS error and relative RMS with altitude for the eight different tomographic schemes. In general, the RMS errors follow an increase-decrease pattern with the increase in altitude, which is in accordance with the IED variation with altitude. For the best tomographic scheme Para_25, its RMS error generally increases to $2.50 \times 10^{5} \mathrm{el} / \mathrm{cm}^{3}$ at the altitude of about $320 \mathrm{~km}$, and then it decreases to $0.50 \times 10^{5} \mathrm{el} / \mathrm{cm}^{3}$ at the uppermost layer (about $1000 \mathrm{~km}$ ). In terms of the relative RMS, its value decreases from $71 \%$ at the lowest layer to $25 \%$ at the altitude of $360 \mathrm{~km}$ followed by an increase to about $80 \%$ at the upper layers $(>800 \mathrm{~km})$, revealing the deficiency of tomography in 
Fig. 7 RMS errors (left panel) and relative RMS errors (right panel) of the differences between IED derived from ionosonde and tomography on different altitude layers during June 1-30, 2015

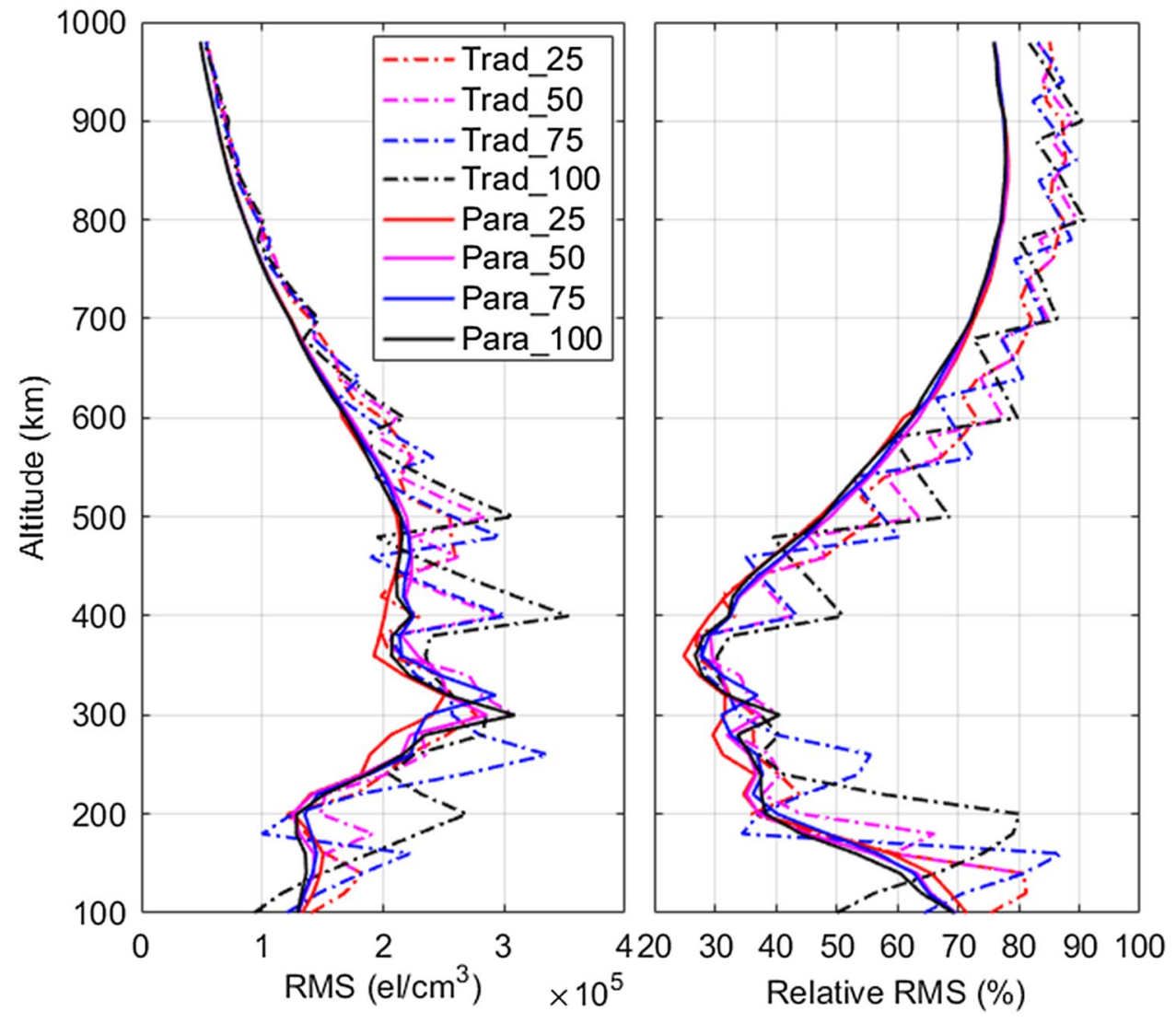

retrieving the IED of high-altitude layers. Generally speaking, tomographic IED fields solved by parameterized method (curve with hollow square) are better than those derived by traditional method (curve with solid triangle) at most layers. The zigzag changes of the RMS and relative RMS shown by the traditional schemes are caused by the improper assumption of homogeneous IED distribution within each voxel. In addition, the tomographic scheme of Para_25 obtains the best performance, which is consistent with the evaluation shown in the above section using GPS data. Refer to Figs. 5 and 6 again, IED profiles derived from Trad_25 and Para_25 are similar, while large discrepancies also occur. We are unable to make sure which profiles are closer to the truths, but we are confident that Para_25 performs better than Trad_25 as validated by both GNSS and ionosonde data.

\section{Comparison of IEDs with Swarm}

The main deficiency of the above two assessments is the limited spatial coverage of the reference datasets. Langmuir probes onboard the Swarm satellites can measure the in situ IED from a global scale, offering an opportunity to validate our tomographic results from the perspective of spatial coverage. Swarm, a European Space Agency mission, consists of three identical satellites to study the earth's magnetic field (Olsen et al. 2013). The Swarm A and C satellites are flying side by side at the same altitude of about $450 \mathrm{~km}$ and the Swarm B satellite is placed at an orbit of about $510 \mathrm{~km}$ (Pignalberi et al. 2016). To further validate the tomographic IEDs, we adopted the Langmuir data from the three Swarm satellites from June 1-30, 2015 as a reference. As shown in Fig. 8, the footprints of the Swarm satellites over the studied period almost have complete coverage of the tomographic area. Thus, it is possible to exploit Swarm measurements to perform a comprehensive assessment on the tomographic solutions in terms of spatial coverage.

Figure 9 displays the comparisons of IED between Swarm and tomography. Basically, IEDs retrieved by the tomography agree well with the Swarm observed ones for all the eight schemes. Compared with the traditional schemes shown in the left four panels, parameterized schemes shown in the right four panels are less likely to have large discrepancies. Though IEDs solved by the scheme of Trad_75 show a very good agreement with Swarm IEDs as their regression line is very close to $1: 1$ line, a considerable number of large discrepancies is also observed. To quantify their performance, statistics of bias, RMS error and correlation coefficient are given in Table 2. The biases of the eight schemes are all positive, suggesting an underestimation of $2-6 \times 10^{4} \mathrm{el} / \mathrm{cm}^{3}$ for tomographic IEDs to Swarm. In addition, the RMS errors of the IED differences between Swarm and the four traditional schemes vary in 
Fig. 8 Footprints of the Swarm satellites over the tomographic region during the period of June $1-30,2015$

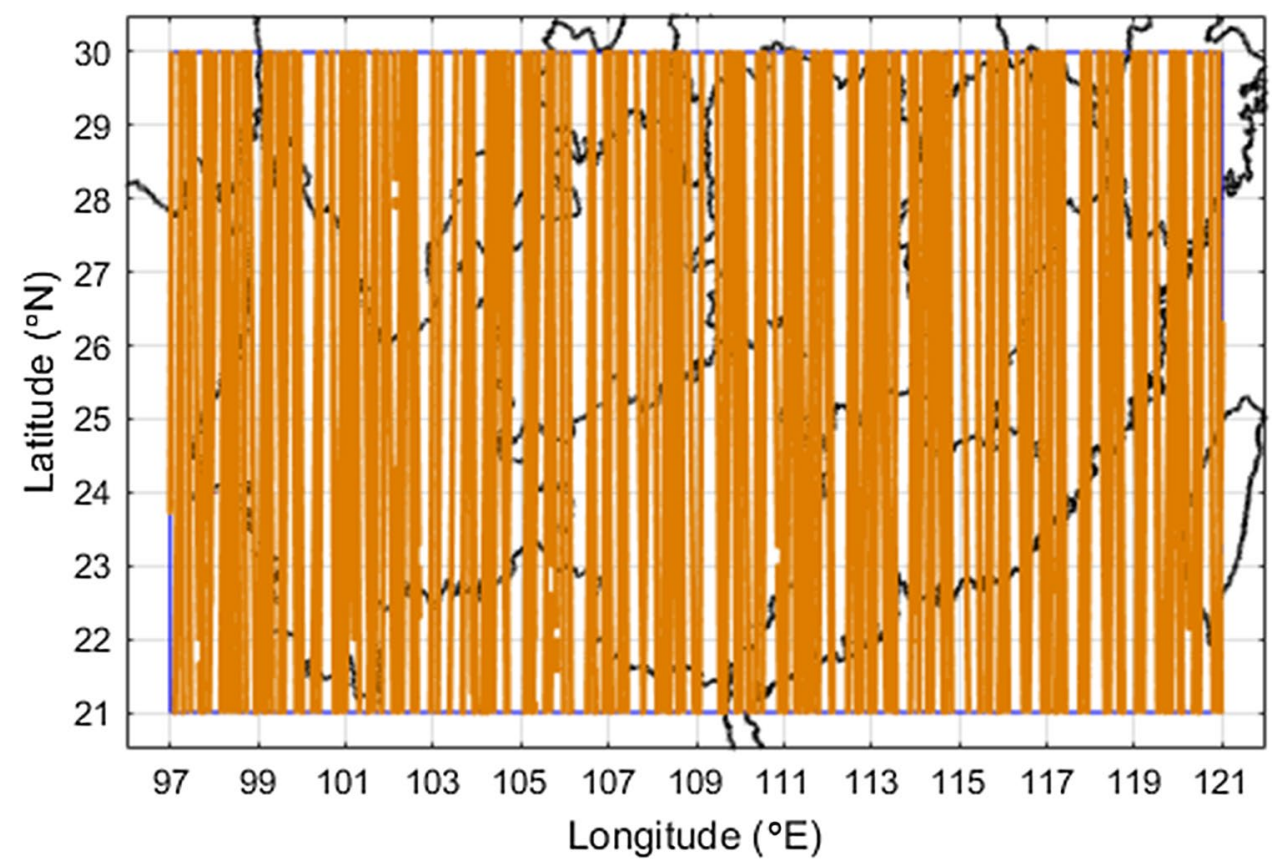

the range of $1.61-1.71 \times 10^{5} \mathrm{el} / \mathrm{cm}^{3}$. For the parameterized method, RMS errors of $1.32 \times 10^{5}, 1.29 \times 10^{5}, 1.34 \times 10^{5}$ and $1.36 \times 10^{5} \mathrm{el} / \mathrm{cm}^{3}$ are obtained for Para_25, Para_50, Para_75 and Para_100, respectively. Compared with the traditional four schemes, the respective improvements are $20 \%, 24 \%, 22 \%$ and $16 \%$. As given in the last column of Table 2, a high correlation coefficient of values greater than 0.8 is achieved by all eight schemes. The highest correlation coefficient is obtained by Para_50 with a value of 0.894 , and comparable correlation coefficients are achieved by the other three parameterized schemes. One may note that in this evaluation the best performance is obtained by the Para_50, which is not consistent with the results from the above two assessments. In this comparison, the Para 25 has the second-best performance and its statistics are very close to those of Para_50. Actually, in the previous two assessments, Para_25 and Para_50 are also very comparable in performance. Therefore, it is not strange that Para_50 shows slightly better agreement than Para_25 in comparison with Swarm. We can still conclude that Para_25 has the overall best performance among the eight tomographic schemes.

\section{Conclusions and outlook}

We presented a new parameterized method for refining the ionospheric tomography modeling and demonstrated its ability to reconstruct the IED field with high quality. In this method, IEDs within a voxel are no longer taken as homogeneous distribution but are regarded to be changeable in space. For any point, its IED is determined via vertically exponential and horizontally inverse distance weighted interpolations from the eight IED values at the corners of the voxel, in which the point is located. To assess the performance of the parameterized model, tomographic experiments were performed using STEC data collected from 45 GPS stations of south China over the period of June 1-30, 2015. Four different vertical resolutions, i.e., $25 \mathrm{~km}, 50 \mathrm{~km}$, $75 \mathrm{~km}$ and $100 \mathrm{~km}$, were tested to examine their impacts on the tomography.

Tomographic solutions were assessed with ionospheric data derived from GPS, ionosonde and Swarm satellites. All assessments indicated superior performance of the parameterized method over the traditional method (i.e., IEDs are assumed to be constant within each voxel). For instance, in the evaluation by Swarm data, the parameterized method outperformed the traditional method by $20 \%, 24 \%, 22 \%$ and $16 \%$ for vertical resolutions of $25,50,75$ and $100 \mathrm{~km}$, respectively. It showed that the scheme Para 25 (parameterized method using a vertical resolution of $25 \mathrm{~km}$ ) achieves the overall best performance. Especially, the Para_25 scheme has shown the following performance: (1) tomographic STECs achieved an accuracy 2.84 TECU when assessed by GPS inferred STEC measurements; (2) in the evaluation of IED profiles by ionosonde, the RMS errors follow an increase-decrease change, from $1.40 \times 10^{5} \mathrm{el} / \mathrm{cm}^{3}$ at the altitude of $100 \mathrm{~km}$ to $2.50 \times 10^{5} \mathrm{el} / \mathrm{cm}^{3}$ at the $\mathrm{F} 2$ layer of about $320 \mathrm{~km}$, and then to $0.50 \times 10^{5} \mathrm{el} / \mathrm{cm}^{3}$ at the uppermost layer of about $1000 \mathrm{~km}$; (3) the tomographic results yield an RMS error of $1.32 \times 10^{5} \mathrm{el} / \mathrm{cm}^{3}$ and a high correlation coefficient of 0.888 when assessed by the Swarm measured in situ IEDs. 
Fig. 9 Scatter plots of tomographic IEDs versus Swarm IEDs for the eight schemes

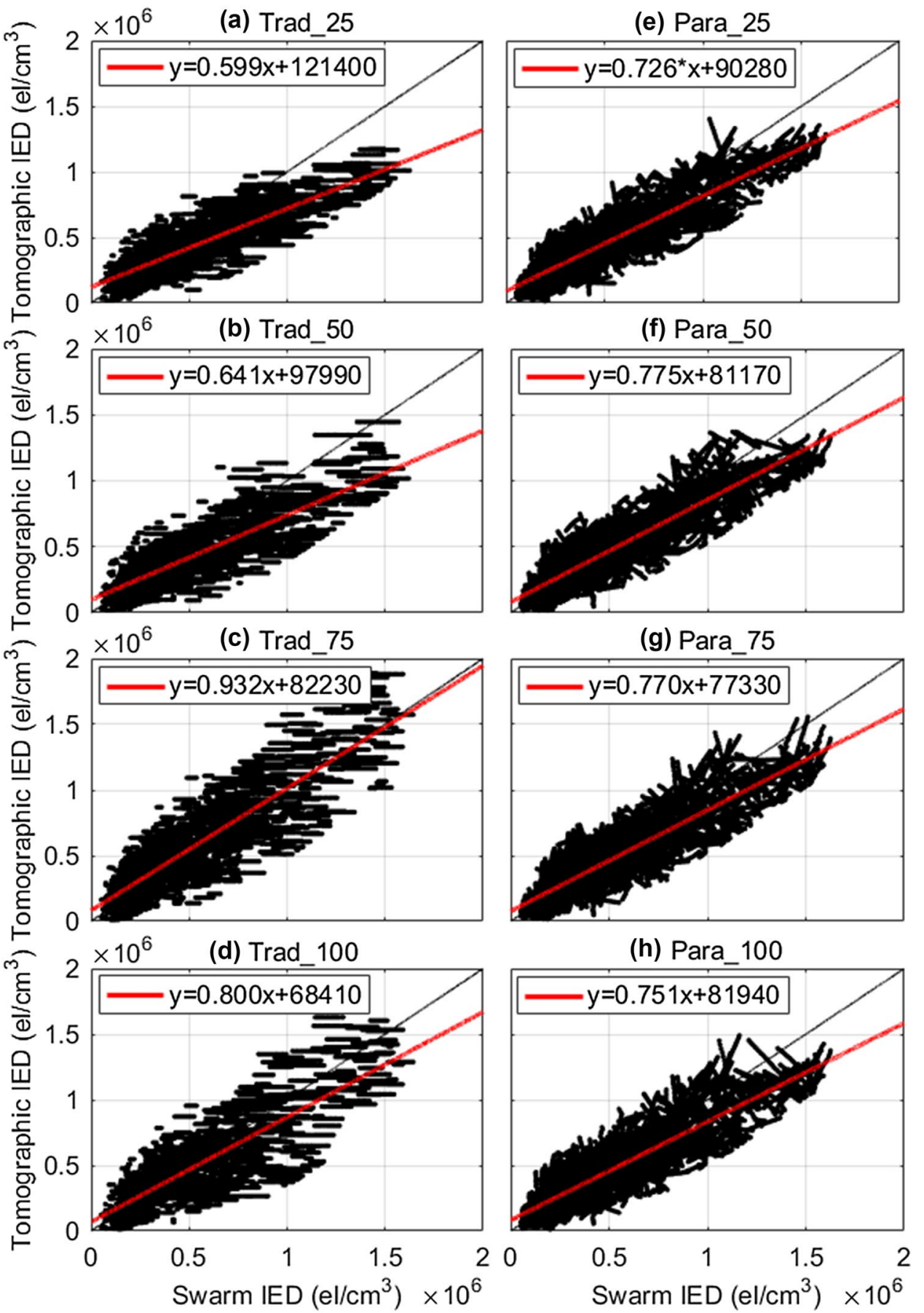

This work demonstrated the potentials of retrieving high accuracy IED fields using the proposed parameterized ionospheric tomography. Due to the advantage of avoiding time-consuming matrix operations, especially for tomography with a large number of voxels, MART solution is used as a representative of all voxel-based methods in the implementation of the parameterized ionospheric tomography. Nevertheless, it is also suggested to apply other inversion methods such as Tikhonov regularization, Bayesian statistical inversion and Singular Value Decomposition to examine their benefits to the parameterized tomographic solution. In addition, future research will focus on the three following issues: (1) examining the performance of the parameterized tomography in other regions with highly dynamic ionosphere, e.g. equatorial regions; (2) developing a strategy to improve the reliability of tomographic IED fields with higher temporal resolutions (e.g. $15 \mathrm{~min}$ ); and (3) investigating the IED anomalies before and during the occurrence of geohazards such as earthquake and volcano eruption. 
Table 2 Statistical results of the differences between IEDs measured by Swarm satellites and IEDs derived from tomographic solutions using traditional and parameterized methods

\begin{tabular}{llll}
\hline Method & Bias $\left(\mathrm{el} / \mathrm{cm}^{3}\right)$ & $\mathrm{RMS}\left(\mathrm{el} / \mathrm{cm}^{3}\right)$ & $\begin{array}{l}\text { Correlation } \\
\text { coefficient }\end{array}$ \\
\hline Trad_25 & $5.49 \times 10^{4}$ & $1.65 \times 10^{5}$ & 0.844 \\
Trad_50 & $5.99 \times 10^{4}$ & $1.69 \times 10^{5}$ & 0.833 \\
Trad_75 & $5.17 \times 10^{4}$ & $1.71 \times 10^{5}$ & 0.861 \\
Trad_100 & $2.04 \times 10^{4}$ & $1.61 \times 10^{5}$ & 0.841 \\
Para_25 & $2.01 \times 10^{4}$ & $1.32 \times 10^{5}$ & 0.888 \\
Para_50 & $1.80 \times 10^{4}$ & $1.29 \times 10^{5}$ & 0.894 \\
Para_75 & $2.40 \times 10^{4}$ & $1.34 \times 10^{5}$ & 0.887 \\
Para_100 & $2.80 \times 10^{4}$ & $1.36 \times 10^{5}$ & 0.884 \\
\hline
\end{tabular}

Statistics are derived using datasets covering the whole month of June 2015

Acknowledgements This work was supported by the National Key R\&D Program of China (Grant No. 2018YFC1503501) and Research Grant for Specially Hired Associate Professor of Central South University (Project No. 202045005). Ying Xu thanks grant support from the National Natural Science Foundation of China (Project No. 41704021). The authors would like to thank the data center of the Crustal Movement Observation Network of China for providing GPS data. We also thank Prof. Baiqi Ning and Dr. Xiukuan Zhao of the Institute of Geology and Geophysics, Chinese Academy of Sciences are appreciated for providing the ionosonde data, via the Data Center for Geophysics, Data Sharing Infrastructure of Earth System Science. The Swarm ionospheric electron density data were provided by the European Space Agency, from the website https://swarm-diss.eo.esa.int/.

Open Access This article is distributed under the terms of the Creative Commons Attribution 4.0 International License (http://creativeco mmons.org/licenses/by/4.0/), which permits unrestricted use, distribution, and reproduction in any medium, provided you give appropriate credit to the original author(s) and the source, provide a link to the Creative Commons license, and indicate if changes were made.

\section{References}

Alizadeh MM, Schuh H, Schmidt M (2015) Ray tracing technique for global 3-D modeling of ionospheric electron density using GNSS measurements. Radio Sci 50:539-553. https://doi. org/10.1002/2014RS005466

Andersen AH, Kak AC (1984) Simultaneous Algebraic Reconstruction Technique (SART): a superior implementation of the ART algorithm. Ultrason Imaging 6:81-94

Austen JR, Franke SJ, Liu CH, Yeh KC (1986) Application of computerized tomography technique to ionospheric research. In: Tauriainen A (ed) URSI and COSPAR international beacon satellite symposium on radio beacon contribution to the study of ionization and dynamics of the ionosphere and to corrections to geodesy and technical workshop, Oulu, Finland. Proc Part 1, 25., University of Oulu, ISBN 951-42-2256-3

Bender M, Dick G, Ge M, Deng Z, Wickert J, Kahle HG, Raabe A, Tetzlaff G (2011) Development of a GNSS water vapour tomography system using algebraic reconstruction techniques. Adv Space Res 47(10):1704-1720. https://doi.org/10.1016/j.asr.2010.05.034
Bhuyan K, Singh SB, Bhuyan PK (2002) Tomographic reconstruction of the ionosphere using generalized singular value decomposition. Curr Sci 83(9): 1117-1120

Bidaine B, Warnant R (2010) Assessment of the NeQuick model at mid-latitudes using GNSS TEC and ionosonde data. Adv Space Res 45(9):1122-1128. https://doi.org/10.1016/j.asr.2009.10.010

Bust GS (2004) Ionospheric data assimilation three-dimensional (IDA3D): a global, multisensor, electron density specification algorithm. J Geophys Res. https://doi.org/10.1029/2003ja010234

Bust GS, Mitchell CN (2008) History, current state, and future directions of ionospheric imaging. Rev Geophys 46(1):1-23. https:// doi.org/10.1029/2006RG000212

Chen B, Liu Z (2014) Voxel-optimized regional water vapor tomography and comparison with radiosonde and numerical weather model. J Geod 88(7):691-703. https://doi.org/10.1007/s0019 0-014-0715-y

Ding N, Zhang S, Zhang Q (2017) New parameterized model for GPS water vapor tomography. Ann Geophys 35(2):311-323. https:// doi.org/10.5194/angeo-35-311-2017

dos Santos Prol F, de Oliveira Camargo P (2015) Ionospheric tomography using GNSS: multiplicative algebraic reconstruction technique applied to the area of Brazil. GPS Solut 20(4):807-814. https://doi.org/10.1007/s10291-015-0490-0

Gao Y, Liu Z (2002) Precise ionosphere modeling using regional GPS network data. J Glob Position Syst 1(1):18-24

Habarulema JB, Carelse SA (2016) Long-term analysis between radio occultation and ionosonde peak electron density and height during geomagnetic storms. Geophys Res Lett 43(9):4106-4111. https:// doi.org/10.1002/2016GL068944

Hansen AJ, Walter T, Enge P (1997) Ionospheric correction using tomography. In: Proceedings of the ION GPS 1997, Institute of Navigation, Kansas City, Missouri, Tennessee, USA, September 16-19, pp 247-257

He L, Heki K, Wu L (2018) Three-dimensional and trans-hemispheric changes in ionospheric electron density caused by the great solar eclipse in North America on 21 August 2017. Geophys Res Lett 45(20):10933-10940. https://doi.org/10.1029/2018g1080365

Hirahara K (2000) Local GPS tropospheric tomography. Earth Planets Space 52:935-939

Jin S, Li D (2018) 3-D ionospheric tomography from dense GNSS observations based on an improved two-step iterative algorithm. Adv Space Res 62(4):809-820. https://doi.org/10.1016/j. asr.2018.05.032

Jin S, Park J-U (2007) GPS ionospheric tomography: a comparison with the IRI-2001 model over South Korea. Earth Planets Space 59(4):287-292

Kersley L (2005) Ionospheric tomography and its applications in radio science and geophysical investigations. Ann Geophys 43(8):535-548

Komjathy A (1997) Global ionospheric total electron content mapping using the global positioning system. Dissertation, University of New Brunswick

Ma XF (2005) Three-dimensional ionospheric tomography using observation data of GPS ground receivers and ionosonde by neural network. J Geophys Res 110(A05308):1-12. https://doi. org/10.1029/2004JA010797

Minkwitz D, van den Boogaart KG, Gerzen T, Hoque M (2015) Tomography of the ionospheric electron density with geostatistical inversion. Ann Geophys 33:1071-1079. https://doi. org/10.5194/angeo-33-1071-2015

Minkwitz D, van den Boogaart KG, Gerzen T et al (2016) Ionospheric tomography by gradient-enhanced kriging with STEC measurements and ionosonde characteristics. Ann Geophys 34:999-1010. https://doi.org/10.5194/angeo-34-999-2016 
Nesterov IA, Kunitsyn VE (2011) GNSS radio tomography of the ionosphere: the problem with essentially incomplete data. Adv Space Res 47(10):1789-1803. https://doi.org/10.1016/j.asr.2010.11.034

Nilsson T, Gradinarsky L (2006) Water vapor tomography using GPS phase observations: simulation results. IEEE Trans Geosci Remote Sens 44:2927-2941. https://doi.org/10.1109/TGRS.2006.877755

Norberg J, Roininen L, Vierinen J et al (2015) Ionospheric tomography in Bayesian framework with Gaussian Markov random field priors. Radio Sci 50:138-152. https://doi.org/10.1002/2014RS0054 31

Norberg J, Vierinen J, Roininen L et al (2018) Gaussian Markov random field priors in ionospheric 3-D multi-instrument tomography. IEEE Trans Geosci Remote Sens 56:7009-7021. https://doi. org/10.1109/TGRS.2018.2847026

Olsen N, Friis-Christensen E, Floberghagen R et al (2013) The Swarm Satellite Constellation Application and Research Facility (SCARF) and Swarm data products. Earth Planets Space 65(11):1189-1200. https://doi.org/10.5047/eps.2013.07.001

Perler D, Geiger A, Hurter F (2011) 4D GPS water vapor tomography: new parameterized approaches. J Geod 85(5):539-550. https:// doi.org/10.1007/s00190-011-0454-2

Pignalberi A, Pezzopane M, Tozzi R et al (2016) Comparison between IRI and preliminary Swarm Langmuir probe measurements during the St. Patrick storm period. Earth Planets Space 68(1):1-18. https ://doi.org/10.1186/s40623-016-0466-5

Pulinets S, Boyarchuk K (2004) Ionospheric precursors of earthquakes. Springer, New York

Reinisch BW, Galkin IA, Khmyrov GM, Kozlov AV, Bibl K, Lisysyan IA, Cheney GP, Huang X, Kitrosser DF, Paznukhov VV, Luo Y, Jones W, Stelmash S, Hamel R, Grochmal J (2009) New Digisonde for research and monitoring applications. Radio Sci 44(1):1-15. https://doi.org/10.1029/2008RS004115

Rius A, Ruffini G, Cucurull L (1997) Improving the vertical resolution of ionospheric tomography with GPS occultations. Geophys Res Lett 24(18):2291-2294

Ruffini G, Flores A, Rius A (1998) GPS tomography of the ionospheric electron content with a correlation functional. IEEE Trans Geosci Remote Sens 36(1):143-153

Seemala GK, Yamamoto M, Saito A, Chen C-H (2014) Three-dimensional GPS ionospheric tomography over Japan using constrained least squares. J Geophys Res Space Phys 119:3044-3052. https:// doi.org/10.1002/2013JA019582

Wang S, Huang S, Xiang J, Fang H, Feng J, Wang Y (2016) Threedimensional ionospheric tomography reconstruction using the model function approach in Tikhonov regularization. J Geophys Res 121:12104-12115. https://doi.org/10.1002/2016JA023487

Wen D, Yuan Y, Ou J, Zhang K, Liu K (2008) A hybrid reconstruction algorithm for 3-D ionospheric tomography. IEEE Trans Geosci Remote Sens 46(6):1733-1739. https://doi.org/10.1109/ TGRS.2008.916466

Wen D, Wang Y, Norman R (2012) A new two-step algorithm for ionospheric tomography solution. GPS Solut 16(1):89-94. https ://doi.org/10.1007/s10291-011-0211-2

Yang Z, Song S, Jiao W, Chen G, Xue J, Zhou W, Zhu W (2017) Ionospheric tomography based on GNSS observations of the CMONOC: performance in the topside ionosphere. GPS Solut 21:363-375. https://doi.org/10.1007/s10291-016-0526-0

Yao Y, Chen P, Zhang S, Chen J (2013a) A new ionospheric tomography model combining pixel-based and function-based models. Adv Space Res 52:614-621. https://doi.org/10.1016/j. asr.2013.05.003

Yao Y, Tang J, Kong J, Zhang L, Zhang S (2013b) Application of hybrid regularization method for tomographic reconstruction of midlatitude ionospheric electron density. Adv Space Res 52:22152225. https://doi.org/10.1016/j.asr.2013.09.030
Yao Y, Tang J, Chen P, Zhang S, Chen J (2014) An improved iterative algorithm for 3-D ionospheric tomography reconstruction. IEEE Trans Geosci Remote Sens 52(2):4696-4706. https://doi. org/10.1109/TGRS.2013.2283736

Zheng D, Hu W, Nie W (2015) Multiscale ionospheric tomography. GPS Solut 19:579-588. https://doi.org/10.1007/s1029 1-014-0418-0

Zheng D, Zheng H, Wang Y, Nie W, Li C, Ao M, Hu W, Zhou W (2017) Variable pixel size ionospheric tomography. Adv Space Res 59(12):2969-2986. https://doi.org/10.1016/j.asr.2017.03.031

Publisher's Note Springer Nature remains neutral with regard to jurisdictional claims in published maps and institutional affiliations.

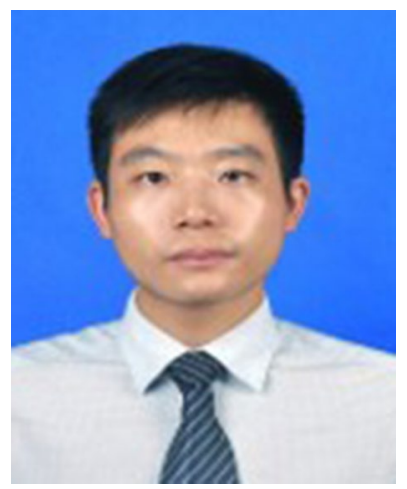

Biyan Chen is currently an associate professor at Central South University. He received his Ph.D. degree from the Hong Kong Polytechnic University in 2017. His research interests include GNSS meteorology, water vapor tomography, extreme weather forecasting and seismo-ionospheric anomalies.

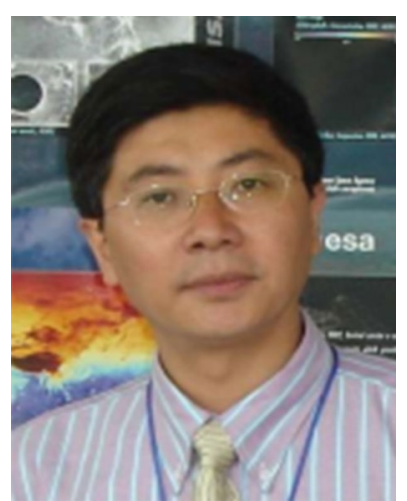

Lixin Wu is currently a leading professor at the School of Geosciences and Info-Physics of the Central South University, China. He received his Ph.D. from the China University of Mining and Technology in 1997. His research mainly focuses on geohazard cognition and perception, $3 \mathrm{D}$ geological modeling and its applications, remote sensing rock mechanics.

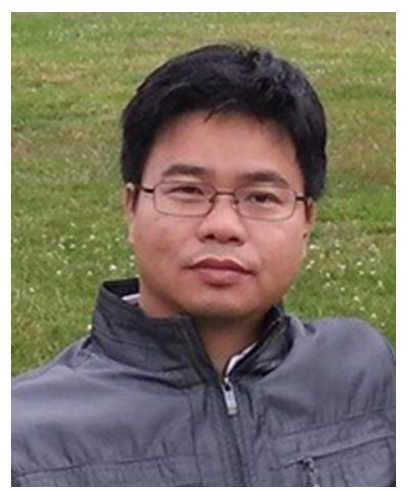

Wujiao Dai is a professor in the School of Geosciences and InfoPhysics at the Central South University, China. He received his Ph.D. from Central South University in 2007 and worked as a research assistant in Hong Kong Polytechnic University for 3 years. His research mainly focuses on GNSS data processing and deformation monitoring. 


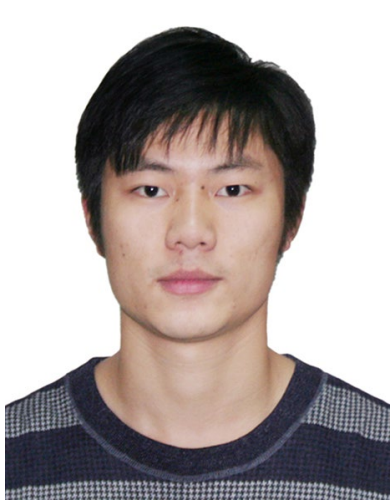

Xiaomin Luo is currently a Ph.D. student at GNSS Research Center, Wuhan University. He received his Master's degree from the Central South University in 2014. His current research mainly focuses on GNSS ionospheric scintillation and GNSS PPP.

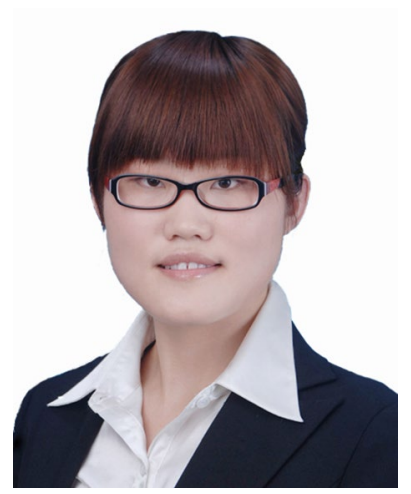

Ying $\mathrm{Xu}$ is a lecturer in the College of Geomatics, Shandong University of Science and Technology, Qingdao, China. She received her $\mathrm{Ph}$.D. from the Hong Kong Polytechnic University in 2016. Her research interests include GNSS data processing and tropospheric modeling. 